\title{
AV-Fistula or Tunnelled Catheter for Haemodialysis?
}

\section{Thomas Ryzlewicz*}

Dialysis Centre, ViaMedis Riesa, Germany

\section{Editorial}

When Haemodialysis started by Kolff and Alwall in the forties, it was a long way of two and a half decade, to transfer this new treatment to the first regular CKD-5 Patients. The lack of the vascular access was the one and only reason. To start, the first access was the Scribner-Quinton Shunt, but this was more or less a makeshift. In 1962 James Cimino published, that Haemodialysis could be done using two different veins(!). Kenneth Appel (together with James Cimino) had sewed in 1966 the first $3 \mathrm{~mm}$ anastomosis between an artery and a superficial vein, that later on was named Cimino-Brescia-Fistula. This invention of this vascular access (together with the design of a single-pass dialysis generator (S. Shaldon) using Acetate concentrate) had shifted the trials of Dialysis into a regular clinical treatment. The final version of the Cimino-Fistula had done in 1967 (side to end anastomosis), so the Haemodialysis had spread all over in the world.

In 1988 Francis (Royal Free, London) had combined Shaldon's Catheter with the top of a Tenckhoff Catheter for a patient planed for living donor transplantation in order to bridge a half year Haemodialysis treatment. This catheter of Francis had tunnelled, the tip had placed in the right atrium of the heart. There was total sterile handling. After six month, the living donor transplantation had done and the Francis Catheter had taken off. Learning from this: There was total sterile handling and a limited time of treatment.

The problem of this decade concerning the Vascular Access for CKD-5 Patients? It is the wide spread use of tunnelled Dialysis Catheters (up to $70 \%$ in the US (>USRDS ESRD Database)) in comparison to

the classical Cimino-Fistula (19\%). The preference Dialysis Catheters results in reasons of infection in 30\% of hospitalization. Easily an infection of a Dialysis Catheter can shift to a life threating complication, the sepsis. The wide spread use of Dialysis Catheters contradicts all serous recommendations of KDOQI/NKF Clinical Practice Guidelines, EDTA Best Practice Guidelines and of DOPPS Report concerning a vascular access for CKD-5 Patients. The Catheter Sepsis can shorten Patient's life seriously. An infection of a Cimino-Fistula remains a local problem and - as there is no plastic - there is save handling.

Narender Goel had clearly shown, that in his study there was no referral from the nephrologists to the vascular surgeons to create a Cimino-Fistula or a PTFE graft. These patients in state of CKD-4 had treated by the nephrologists. But reaching the state of CKD-5, the majority of them had no vascular access! When you tell this context to a layman, he never would believe this! But this is clinical reality. Why can a bigger part of nephrologists neglect continuously these mentioned recommendations?

This behaviour of the prevalence of Dialysis Catheters as the Vascular Access is a serious point. It shows: The medical education does not reach an important part of nephrologists. So the Dialysis Providers will kindly asked to solve this problem. With a classical Cimino-Fistula, there is very low risk of infection. When there is a better quality of treatment (>this means here without a Catheter Infection), there is longer living of the patients. If the ethical reasons to improve the quality of treatment to reach an av-fistula for the majority of the CKD-5 Patients are not sufficient, perhaps the Providers of the Dialysis Centres can help to improve the quality of treatment.
*Corresponding author: Thomas Ryzlewicz, ViaMedis Kidney Centre, RobertKoch-Strasse 30, D-01589, Riesa, Germany, Tel: +49 17283666 25; E-mail: thomas.ryzlewicz@web.de

Received November 27, 2015; Accepted November 28, 2015; Published December 07, 2015

Citation: Ryzlewicz T (2015) AV-Fistula or Tunnelled Catheter for Haemodialysis? J Kidney 1: e102. doi:10.4172/2472-1220.1000e102

Copyright: @ 2015 Ryzlewicz T. This is an open-access article distributed under the terms of the Creative Commons Attribution License, which permits unrestricted use, distribution, and reproduction in any medium, provided the original author and source are credited. 